\title{
Constantin's inequality for nabla and diamond-alpha derivative
}

\author{
Ayşe Feza Güvenilir ${ }^{1 *}$, Billur Kaymakçalan² and Neslihan Nesliye Pelen ${ }^{3}$
}

${ }^{*}$ Correspondence:

guvenili@science.ankara.edu.tr

'Department of Mathematics,

Faculty of Science, Ankara

University, Ankara, Turkey

Full list of author information is

available at the end of the article

\section{Springer}

\begin{abstract}
Calculus for dynamic equations on time scales, which offers a unification of discrete and continuous systems, is a recently developed theory. Our aim is to investigate Constantin's inequality on time scales that is an important tool used in determining some properties of various dynamic equations such as global existence, uniqueness and stability. In this paper, Constantin's inequality is investigated in particular for nabla and diamond-alpha derivatives.
\end{abstract}

Keywords: Constantin's inequality; nabla differentiation; nabla integration; diamond-alpha differentiation; diamond-alpha integration

\section{Introduction}

To study the boundedness of solutions for some nonautonomous second order linear differential equations, Ou-Iang [1] used a nonlinear integral inequality. This type of integral inequality had been also used to obtain global existence, uniqueness and stability properties of various nonlinear differential equations. Pachpatte [2] gave the generalized Ou-Iang type integral inequality. In 1996, Constantin [3] established the following interesting alternative result for this generalization.

Theorem 1.1 Iffor some $k, T>0, u \in C\left(\mathbb{R}_{0}^{+}, \mathbb{R}_{0}^{+}\right)$satisfies

$$
u^{2}(t) \leq k^{2}+2 \int_{0}^{t}\left\{f(s) u(s)\left[u(s)+\int_{0}^{s} g(\tau) w(u(\tau)) d \tau\right]+h(s) u(s)\right\} d s,
$$

$\forall t \in[0, T]$, where $f, g, h \in C\left(\mathbb{R}_{0}^{+}, \mathbb{R}_{0}^{+}\right)$and w belongs to the class of continuous nondecreasing functions on $\mathbb{R}_{0}^{+}$such that $w(r)>0$ if $r>0$ and satisfies $\int_{1}^{\infty} \frac{d s}{w(s)}=\infty$, then

$$
u(t) \leq K(t)+\int_{0}^{t} f(s) G^{-1}\left\{G(K(t))+\int_{0}^{s}[f(\tau)+g(\tau)] d \tau\right\} d s
$$

where $K(t)=k+\int_{0}^{t} h(s) d s, G(r)=\int_{1}^{r} \frac{d s}{s+w(s)}, r>1 . G^{-1}$ denotes the inverse function of $G$.

Applying above Theorem 1.1 and a topological transversality theorem, he showed that, under some suitable assumptions, the integrodifferential equation

$$
x^{\prime}(t)=F\left(t, x(t), \int_{0}^{t} K[t, s, x(s)] d s\right)
$$

(c) 2015 Güvenilir et al. This article is distributed under the terms of the Creative Commons Attribution 4.0 International License (http://creativecommons.org/licenses/by/4.0/), which permits unrestricted use, distribution, and reproduction in any medium, provided you give appropriate credit to the original author(s) and the source, provide a link to the Creative Commons license, and indicate if changes were made. 
has a solution and gave bounds on that solution. Additionally, Yang and Tan [4] gave the generalization of Constantin's inequality, and they also presented the discrete analogue of this inequality which is stated as follows.

Theorem 1.2 Let the function $w \in C\left(\mathbb{R}_{+}, \mathbb{R}_{+}\right)$be nondecreasing, $w(r)>0$ for $r>0, \phi \in$ $C^{1}\left(\mathbb{R}_{+}, \mathbb{R}_{+}\right)$with $\phi^{\prime}$ being nonnegative and nondecreasing. $u, c \in C\left(\mathbb{N}_{M}, \mathbb{R}_{+}\right)$with $c(n)$ nondecreasing. Further, let

$$
f(n, s), g(n, s), h(n, s) \in C\left(\mathbb{N}_{M} \times \mathbb{N}_{M}, \mathbb{R}_{+}\right)
$$

be nondecreasing with respect to $n$ for every s fixed. Then the discrete inequality

$$
\begin{aligned}
\phi[u(n)] \leq & c(n)+\sum_{s=0}^{n-1}\left\{f(n, s) \phi^{\prime}[u(s)] \times\left[u(s)+\sum_{\xi=0}^{s-1} g(s, \xi) w(u(\xi))\right]\right. \\
& \left.+h(n, s) \phi^{\prime}[u(s)]\right\}, \quad n \in \mathbb{N}_{M}
\end{aligned}
$$

implies

$$
u(n) \leq L(n)+\sum_{s=0}^{n-1} f(n, s) G^{-1}\left(G[L(n)]+\sum_{\xi=0}^{s-1} f(n, \xi)+g(n, \xi)\right) .
$$

Here $G(r)=\int_{r_{0}}^{r} \frac{d s}{s+w(s)}, r \leq r_{0}, 1>r_{0}>0, \lim _{x \rightarrow \infty} G(x)=\infty, L(n)=\phi^{-1}[c(n)]+\sum_{s=0}^{n-1} h(n, s)$, $\mathbb{N}_{M}=\{n \in \mathbb{N}: n \leq M, M \in \mathbb{N}\}$.

\section{Some basic definitions related to time scales}

Using [5-7] we give the following information. By a time scale, denoted by $\mathbb{T}$, we mean a nonempty closed subset of $\mathbb{R}$. The theory of time scales gives a way to unify continuous and discrete analysis.

The set $\mathbb{T}^{\kappa}$ is defined by $\mathbb{T}^{\kappa}=\mathbb{T} /(\rho(\sup \mathbb{T})$, sup $\mathbb{T}]$ and the set $\mathbb{T}_{\kappa}$ is defined by $\mathbb{T}_{\kappa}=$ $\mathbb{T} /[\inf \mathbb{T}, \sigma(\inf \mathbb{T}))$. The forward jump operator $\sigma: \mathbb{T} \rightarrow \mathbb{T}$ is defined by $\sigma(t):=\inf (t, \infty)_{\mathbb{T}}$, for $t \in \mathbb{T}$. The backward jump operator $\rho: \mathbb{T} \rightarrow \mathbb{T}$ is defined by $\rho(t):=\sup (-\infty, t)_{\mathbb{T}}$, for $t \in \mathbb{T}$. The forward graininess function $\mu: \mathbb{T} \rightarrow \mathbb{R}_{0}^{+}$is defined by $\mu(t):=\sigma(t)-t$, for $t \in \mathbb{T}$. The backward graininess function $v: \mathbb{T} \rightarrow \mathbb{R}_{0}^{+}$is defined by $v(t):=t-\rho(t)$, for $t \in \mathbb{T}$. Here it is assumed that $\inf \emptyset=\sup \mathbb{T}$ and $\sup \emptyset=\inf \mathbb{T}$.

For a function $f: \mathbb{T} \rightarrow \mathbb{T}$, we define the $\Delta$-derivative of $f$ at $t \in \mathbb{T}^{\kappa}$, denoted by $f^{\Delta}(t)$ for all $\epsilon>0$. There exists a neighborhood $U \subset \mathbb{T}$ of $t \in \mathbb{T}^{\kappa}$ such that

$$
\left|f(\sigma(t))-f(s)-f^{\Delta}(t)(\sigma(t)-s)\right| \leq \epsilon|\sigma(t)-s|
$$

for all $s \in U$.

For the same function define the $\nabla$-derivative of $f$ at $t \in \mathbb{T}_{\kappa}$, denoted by $f^{\nabla}(t)$, for all $\epsilon>0$. There exists a neighborhood $V \subset \mathbb{T}$ of $t \in \mathbb{T}_{\kappa}$ such that

$$
\left|f(s)-f(\rho(t))-f^{\nabla}(t)(s-\rho(t))\right| \leq \epsilon|s-\rho(t)|
$$

for all $s \in V$. 
We define $\diamond_{\alpha}$-derivative of $f$ at $t \in \mathbb{T}_{\kappa}^{\kappa}$, denoted by $f^{\diamond_{\alpha}}(t)$ for all $\epsilon>0$. There is a neighborhood $U \subset \mathbb{T}$ such that for any $s \in U$,

$$
\begin{aligned}
& |\alpha| f(\sigma(t))-f(s)|| \rho(t)-s|+(1-\alpha)| f(\rho(t))-f(s)|| \sigma(t)-s\left|-f^{\diamond_{\alpha}}(t)\right| \rho(t)-s|| \sigma(t)-s|| \\
& \quad \leq \epsilon|\rho(t)-s||\sigma(t)-s| .
\end{aligned}
$$

A function $f: \mathbb{T} \rightarrow \mathbb{R}$ is rd-continuous if it is continuous at right-dense points in $\mathbb{T}$ and its left-sided limits exist at left-dense points in $\mathbb{T}$. The class of real rd-continuous functions defined on a time scale $\mathbb{T}$ is denoted by $C_{\mathrm{rd}}(\mathbb{T}, \mathbb{R})$. If $f \in C_{\mathrm{rd}}(\mathbb{T}, \mathbb{R})$, then there exists a function $F(t)$ such that $F^{\Delta}(t)=f(t)$. The delta integral is defined by $\int_{a}^{b} f(x) \Delta x=F(b)-F(a)$.

Similarly, a function $g: \mathbb{T} \rightarrow \mathbb{R}$ is ld-continuous if it is continuous at left-dense points in $\mathbb{T}$ and its right-sided limits exist at right-dense points in $\mathbb{T}$. The class of real ld-continuous functions defined on a time scale $\mathbb{T}$ is denoted by $C_{\mathrm{ld}}(\mathbb{T}, \mathbb{R})$. If $g \in C_{\mathrm{ld}}(\mathbb{T}, \mathbb{R})$, then there exists a function $G(t)$ such that $G^{\nabla}(t)=g(t)$. The nabla integral is defined by $\int_{a}^{b} g(x) \nabla x=$ $G(b)-G(a)$.

By [8], if a function $h(t): \mathbb{T} \rightarrow \mathbb{R}$ is continuous, then it is diamond-alpha integrable, and the fundamental theorem of calculus is not true for $\diamond_{\alpha}$-derivative. We know that

$$
\left(\int_{a}^{t} h(s) \diamond_{\alpha} s\right)^{\diamond_{\alpha}}=\left(1-2 \alpha+2 \alpha^{2}\right) h(t)+\left(\alpha-\alpha^{2}\right)[h(\rho(t))+h(\sigma(t))] .
$$

Ferreira [9] generalized Constantin's inequality involving delta derivatives on an arbitrary time scale.

Theorem 2.1 Assume that $u \in C_{\mathrm{rd}}\left([a, b]_{\mathbb{T}}, \mathbb{R}_{0}^{+}\right), c \in C_{\mathrm{rd}}\left([a, b]_{\mathbb{T}}, \mathbb{R}^{+}\right)$is nondecreasing, $\Phi \in$ $C\left(\mathbb{R}_{0}^{+}, \mathbb{R}_{0}^{+}\right)$is a strictly increasing function such that

$$
\lim _{x \rightarrow \infty} \Phi(x)=\infty
$$

Let $f(t, \xi), h(t, \xi) \in C_{\mathrm{rd}}\left([a, b]_{\mathbb{T}} \times[a, b]_{\mathbb{T}^{\kappa}}, \mathbb{R}_{0}^{+}\right)$and $g(t, \xi) \in C_{\mathrm{rd}}\left([a, b]_{\mathbb{T}^{\kappa}} \times[a, b]_{\mathbb{T}^{\kappa}}, \mathbb{R}_{0}^{+}\right)$be nondecreasing for every fixed $\xi$. Further, let $w, \phi, \Psi \in C\left(\mathbb{R}_{0}^{+}, \mathbb{R}_{0}^{+}\right)$be nondecreasing such that $\{w, \phi, \Psi\}(x)>0$ for every $x>0$. Define

$$
M(x)=\max \{\phi(x), \Psi(x)\}
$$

on $\mathbb{R}_{0}^{+}$and assume that the following function

$$
F(x)=\int_{x_{0}}^{x} \frac{d s}{M \circ \Phi^{-1}(s)}
$$

with $x>c(a)>x_{0} \geq 0$ if $\int_{0}^{x} \frac{d s}{M_{\circ} \Phi^{-1}(s)}<\infty$ and $x>c(a)>x_{0}>0$ if $\int_{0}^{x} \frac{d s}{M_{\circ} \Phi^{-1}(s)}=\infty$ satisfies $\lim _{x \rightarrow \infty} F(x)=\infty$.

Also assume that the function

$$
P(r)=\int_{r_{0}}^{r} \frac{d s}{w(s)}
$$

where $r \geq 0, r_{0} \geq 0$ if $\int_{0}^{r} \frac{d s}{w(s)}<\infty$ and $r>0, r_{0}>0$ if $\int_{0}^{r} \frac{d s}{w(s)}=\infty$ satisfies $\lim _{r \rightarrow \infty} P(r)=\infty$. 
If $\Phi^{-1}\left[F^{-1}(x)\right] \leq x$, for all $x \geq 0$, the inequality

$$
\Phi[u(t)] \leq c(t)+\int_{a}^{t}\left(f(t, s) \phi[u(s)]\left[u(s)+\int_{a}^{s} g(s, \xi) w[u(s)] \Delta \xi\right]+h(t, s) \Psi[u(s)]\right) \Delta s
$$

for $t \in[a, b]_{T}$ implies

$$
u(t) \leq \Phi^{-1}\left[F^{-1}\left(K(t)+\int_{a}^{t}\left(f(t, s) G^{-1}\left[G(K(t))+\int_{a}^{s} \max \{f(t, \xi), g(t, \xi)\} \Delta \xi\right]\right) \Delta s\right)\right] .
$$

Here $K(t)=: F[c(t)]+\int_{a}^{t} h(t, s) \Delta s$ and $G(r)=\int_{r_{0}}^{r} \frac{d s}{w(s)+s}$.

\section{Some new results}

We try to generalize Constantin's inequality containing nabla and diamond-alpha derivatives and present the results we have obtained.

First we look for the discrete analogue of Constantin's inequality involving nabla derivatives.

Theorem 3.1 Let the function $w \in C\left(\mathbb{R}_{+}, \mathbb{R}_{+}\right)$be nondecreasing, $w(r)>0$ for $r>0, \phi \in$ $C^{1}\left(\mathbb{R}_{+}, \mathbb{R}_{+}\right)$with $\phi^{\prime}$ being nonnegative and nondecreasing. $u, c \in C\left(\mathbb{N}_{M}, \mathbb{R}_{+}\right)$with $c(n)$ nondecreasing. Further, let

$$
f(n, s), g(n, s), h(n, s) \in C\left(\mathbb{N}_{M} \times \mathbb{N}_{M}, \mathbb{R}_{+}\right)
$$

be nondecreasing with respect to $n$ for every s fixed. Then there exist fixed constants $k, l>0$ such that the discrete inequality

$$
\begin{aligned}
\phi[u(n)] \leq & c(n)+\sum_{s=1}^{n}\left\{f(n, s) \phi^{\prime}[u(s)] \times\left[u(s)+\sum_{\xi=1}^{s} g(s, \xi) w(u(\xi))\right]\right. \\
& \left.+h(n, s) \phi^{\prime}[u(s)]\right\}, \quad n \in \mathbb{N}_{M}
\end{aligned}
$$

implies

$$
u(n) \leq L(n)+\sum_{\xi=1}^{n} k f(n, \xi) G^{-1}\left(G[L(n)]+l \sum_{s=1}^{\xi} k f(n, s)+g(n, s)\right)
$$

Here $G(r)=\int_{r_{0}}^{r} \frac{d s}{s+w(s)}, r \leq r_{0}, 1>r_{0}>0, \lim _{x \rightarrow \infty} G(x)=\infty, L(n)=\phi^{-1}[c(n)]+k \sum_{s=1}^{n} h(n, s)$, $\mathbb{N}_{M}=\{n \in N: n \leq M, M \in \mathbb{N}\}$. Assume that $\mathbb{N}$ starts with 1 .

Proof Fixing an arbitrary positive integer $m \in[1, M]$.

We denote the set $J=\{1,2, \ldots, m\}$ and we define a positive function $z(n) \in K\left(J, \mathbb{R}_{+}\right)$such that

$$
z(n)=c(m)+\sum_{s=1}^{n}\left\{f(m, s) \phi^{\prime}[u(s)]\left[u(s)+\sum_{\xi=1}^{s} g(m, \xi) w(u(\xi))\right]+h(m, s) \phi^{\prime}[u(s)]\right\} .
$$


Then $u(n) \leq \phi^{-1}[z(n)]$. Using (1) we get

$$
\frac{\nabla z(n)}{\phi^{\prime}\left[\phi^{-1}[z(n)]\right]} \leq f(m, n)\left[\phi^{-1}[z(n)]+\sum_{s=1}^{n} g(m, s) w\left(\phi^{-1}[z(n)]\right)\right]+h(m, n) .
$$

Since we have finitely many elements in the domain, functions are bounded and, since each of them goes to $\mathbb{R}_{+}$, it never takes 0 . Then

$$
\begin{aligned}
k= & \max \left\{\frac{\phi^{\prime}\left[\phi^{-1}[z(m)]\right]}{\phi^{\prime}\left[\phi^{-1}[z(m-1)]\right]}, \frac{\phi^{\prime}\left[\phi^{-1}[z(m-1)]\right]}{\phi^{\prime}\left[\phi^{-1}[z(m-2)]\right]}, \ldots,\right. \\
& \left.\frac{\phi^{\prime}\left[\phi^{-1}[z(n)]\right]}{\phi^{\prime}\left[\phi^{-1}[z(n-1)]\right]}, \ldots, \frac{\phi^{\prime}\left[\phi^{-1}[z(1)]\right]}{\phi^{\prime}\left[\phi^{-1}[z(0)]\right]}\right\}
\end{aligned}
$$

exists.

If we multiply (2) by $k$ and use the mean value theorem, we get

$$
\nabla \phi^{-1}[z(n)] \leq k f(m, n)\left[\phi^{-1}[z(n)]+\sum_{s=1}^{n} g(m, s) w\left(\phi^{-1}[z(n)]\right)\right]+k h(m, n) .
$$

Substituting $n$ with $\xi$ in the last assertion and summing over $\xi=1, \ldots, n$, we have

$$
\begin{aligned}
\phi^{-1}[z(n)] \leq & \phi^{-1}[z(0)]+k \sum_{\xi=1}^{m} h(m, \xi) \\
& +k \sum_{\xi=1}^{n} f(m, \xi)\left[\phi^{-1}[z(\xi)]+\sum_{s=1}^{\xi} g(m, s) w\left[\phi^{-1}[z(s)]\right]\right] .
\end{aligned}
$$

Define the right-hand side of the last inequality as $v(n)$, then we get $\phi^{-1}[z(n)] \leq v(n)$ for $n \in J$. Taking the nabla derivative of $v(n)$, we obtain

$$
\nabla v(n) \leq k f(m, n)\left[v(n)+\sum_{s=1}^{n} g(m, s) w[v(s)], \quad n \in J\right.
$$

We define $y(n)=v(n)+\sum_{s=1}^{n} g(m, s) w[v(s)]$ and take the nabla derivative of $y(n)$, then we get

$$
\frac{\nabla y(n)}{y(n)+w(y(n))} \leq k f(m, n)+g(m, n)
$$

For inequality (3), substituting $n$ with $s$ and summing over $s=1,2, \ldots, n$, we obtain

$$
\sum_{s=1}^{n} \frac{\nabla y(s)}{y(s)+w(y(s))} \leq \sum_{s=1}^{n} k f(m, s)+g(m, s)
$$

Let $l=\max _{n \in[0, m]} \frac{\sum_{s=1}^{n} \frac{\nabla y(s)}{y(s-1)+w(y(s-1))}}{\sum_{s=1}^{n} \frac{\nabla y(s)}{y(s)+w(y(s))}}$. Multiply (4) by $l$ and by the mean value theorem, we get

$$
y(n) \leq G^{-1}\left(G[y(0)]+l \sum_{s=1}^{n} k f(m, s)+g(m, s)\right) .
$$


Since $\nabla v(n) \leq k f(m, n) y(n)$, we obtain

$$
\nabla v(n) \leq k f(m, n) G^{-1}\left(G[y(0)]+l \sum_{s=1}^{n} k f(m, s)+g(m, s)\right) .
$$

Substituting $n$ with $\xi$ and summing over $\xi=1,2, \ldots, n$ and using $u(n) \leq \phi^{-1}[z(n)] \leq v(n)$, we get

$$
u(n) \leq L(m)+\sum_{\xi=1}^{n} k f(m, \xi) G^{-1}\left(G[L(m)]+l \sum_{s=1}^{\xi} k f(m, s)+g(m, s)\right) .
$$

Hence we get the desired result.

Example 3.1 Let

$$
u^{2}(n)=L+2 \sum_{s=1}^{n} H(n-s) u(s)
$$

Here $n \in[1, M]$. The unique positive solution for equation (5) can be obtained by successive substitution. For instance, by letting $n=1,2$ we obtain

$$
\begin{aligned}
& u(1)=\sqrt{L+2 H(0) u(1)}, \\
& u(2)=\sqrt{L+2 H(1) u(1)+2 H(0) u(2)} .
\end{aligned}
$$

By using solutions of quadratic equations, we can find $u(0), u(1), u(2), \ldots, u(M)$ successively. If we use the theorem above, then the bound for $u$ will be $u(n) \leq \sqrt{L}+k \sum_{s=1}^{n} H(n-$ $s)$. With the help of the proof of Theorem 3.1 here

$$
k=\max _{n \in[1, M]}\left[1+\frac{2 H(M-n) u(n)}{L+2 H(M-1) u(1)+2 H(M-2) u(2)+\cdots+2 H(M-n+1) u(n-1)}\right] .
$$

Here $f(n, s)=g(n, s)=0, h(n, s)=H(n-s), c(n)=L>0, L$ is constant, $u, H \in K\left(\mathbb{N}_{M}, \mathbb{R}_{+}\right), P$ is nondecreasing, and $\phi(x)=x^{2}$.

If we choose $H(n, s), L$ good enough, $k$ does not exceed a number $c$ when $M$ tends to infinity. For instance, if $H(n, s)=1, L=3$, then $k$ does not exceed 3 when $M$ tends to infinity. Therefore, for the equality $u^{2}(n)=3+2 \sum_{s=1}^{n} u(s)$, by using the theorem above, we have the bound for

$$
u(n) \leq \sqrt{3}+3 n, \quad n \in \mathbb{N}
$$

Lemma 3.1 Let $f: \mathbb{R} \rightarrow \mathbb{R}$ be continuously differentiable and $g: \mathbb{T} \rightarrow \mathbb{R}$ be nabla differentiable, then $f \circ g$ is nabla differentiable and the formula is given by

$$
(f \circ g)^{\nabla}(t)=g^{\nabla}(t)\left[\int_{0}^{1} f^{\prime}\left(g(\rho(t))+h v(t) g^{\nabla}(t)\right) d h\right] .
$$

Proof Apply an ordinary substitution rule from calculus and get

$$
f(g(s))-f(g(\rho(t)))=g(s)-g(\rho(t)) \int_{0}^{1} f^{\prime}(h g(s)-(1-h) g(\rho(t))) d h .
$$


Let $t \in \mathbb{T}_{\kappa}$ and $\epsilon>0$ be given. Since $g$ is nabla differentiable at $t$, there exists a neighborhood $U_{1}$ of $t$ such that

$$
\left|g(s)-g(\rho(t))-g^{\nabla}(t)(s-\rho(t))\right| \leq \epsilon^{*}|s-\rho(t)|, \quad \forall s \in U_{1},
$$

where $\epsilon^{*}=\frac{\epsilon}{1+2 \int_{0}^{1}\left|f^{\prime}(h g(s)-(1-h) g(\rho(t)))\right| d h}$.

By assumption we have

$$
\left|f^{\prime}(h(g(s))+(1-h) g(\rho(t)))-f^{\prime}(h(g(t))+(1-h) g(\rho(t)))\right| \leq \frac{\epsilon}{\epsilon^{*}+\left|g^{\nabla}(t)\right|}, \quad \forall s \in U_{2},
$$

where $\forall \delta>0 \exists U_{2}$ is a neighborhood of $t$ such that $|g(s)-g(t)|<\delta, \forall s \in U_{2}$. Here let us define a neighborhood $U$ of $t$ such that $U=U_{1} \cap U_{2}$. Then we have

$$
\begin{aligned}
\left|f \circ g(s)-f \circ g(\rho(t))-(s-\rho(t)) g^{\nabla}(t) \int_{0}^{1} f^{\prime}(\beta) d h\right| \\
=\left|g(s)-g(\rho(t))-(s-\rho(t)) g^{\nabla}(t) \int_{0}^{1} f^{\prime}(\alpha) d h+(s-\rho(t)) g^{\nabla}(t) \int_{0}^{1} f^{\prime}(\alpha)-f^{\prime}(\beta) d h\right| \\
\leq\left|g(s)-g(\rho(t))-(s-\rho(t)) g^{\nabla}(t)\right| \int_{0}^{1}\left|f^{\prime}(\alpha)\right| d h \\
\quad+|s-\rho(t)|\left|g^{\nabla}(t)\right| \int_{0}^{1}\left|f^{\prime}(\alpha)-f^{\prime}(\beta)\right| d h \\
\leq \epsilon^{*}|s-\rho(t)| \int_{0}^{1}\left|f^{\prime}(\alpha)\right| d h+|s-\rho(t)|\left|g^{\nabla}(t)\right| \int_{0}^{1}\left|f^{\prime}(\alpha)-f^{\prime}(\beta)\right| d h \\
\leq \epsilon^{*}|s-\rho(t)| \int_{0}^{1}\left|f^{\prime}(\beta)\right| d h+|s-\rho(t)|\left(\left|g^{\nabla}(t)\right|+\epsilon^{*}\right) \int_{0}^{1}\left|f^{\prime}(\alpha)-f^{\prime}(\beta)\right| d h \\
\leq \\
\leq \frac{\epsilon}{2}|s-\rho(t)|+\frac{\epsilon}{2}|s-\rho(t)|=\epsilon|s-\rho(t)|,
\end{aligned}
$$

where $\alpha=h g(s)+(1-h) g(\rho(t))$ and $\beta=h g(t)+(1-h) g(\rho(t))$. Hence $f \circ g$ is nabla differentiable and its derivative is as claimed above.

This lemma first occurred in the article of Atici et al. [10] as

$$
(f \circ g)^{\nabla}(t)=g^{\nabla}(t)\left[\int_{0}^{1} f^{\prime}\left(g(t)+h v(t) g^{\nabla}(t)\right) d h\right] .
$$

With a counter example, we can show that their version of the formula is not true.

Example 3.2 Let $g(x): \mathbb{Z} \rightarrow \mathbb{R}$ such that $g(n)=\frac{1}{n}, f(x): \mathbb{R} \rightarrow \mathbb{R}$ such that $f(x)=x^{2}$. Therefore the first derivative of $f(x)$ is continuous. If we apply the above formula to find the nabla derivative of the function $(f \circ g)(t)$, we get

$$
\frac{1-2 n}{\left(n^{2}\right)(n-1)^{2}}=\left(\frac{1}{n^{2}}\right)^{\nabla} \neq\left(\frac{1}{n}\right)^{\nabla} \int_{0}^{1}\left[2\left(\frac{1}{n}\right)+2 h\left(\frac{1}{n}\right)^{\nabla}\right] d h=\frac{3-2 n}{\left(n^{2}\right)(n-1)^{2}} .
$$

Lemma 3.2 Let $a, b \in \mathbb{T}$, consider the time scale $[a, b]_{\mathbb{T}}$ and a function $r \in C_{\mathrm{ld}}^{1}\left([a, b]_{\mathbb{T}}, \mathbb{R}\right)$ with $r^{\nabla}(t) \geq 0$. Suppose that a function $g \in C\left(\mathbb{R}_{0}^{+}, \mathbb{R}_{0}^{+}\right)$is positive and nondecreasing on $\mathbb{R}$. 
Then, for each $t \in[a, b]_{\mathbb{T}}$, we have

$$
G(r(t)) \leq G(r(a))+\int_{a}^{t} \frac{r^{\nabla}(\tau)}{g(r(\rho(\tau)))} \nabla \tau
$$

where $G(x)=\int_{x_{0}}^{x} \frac{d s}{g(s)}$, where $x \geq 0, x_{0} \geq 0$ if $\int_{0}^{x} \frac{d s}{g(s)}<\infty$ and $x>0, x_{0}>0$ if $\int_{0}^{x} \frac{d s}{g(s)}=\infty$.

Proof Since $g$ is positive and nondecreasing on $(0, \infty)$, we have

$$
r^{\nabla}(t) \int_{0}^{1} \frac{d h}{g\left(r(\rho(t))+h v(t) r^{\nabla}(t)\right)} \leq \frac{r^{\nabla}(t)}{g(r(\rho(t)))} .
$$

By using Lemma 3.1, we get

$$
(G \circ r)^{\nabla}(t) \leq \frac{r^{\nabla}(t)}{g(r(\rho(t)))}
$$

and

$$
G(r(t)) \leq G(r(a))+\int_{a}^{t} \frac{r^{\nabla}(\tau)}{g(r(\rho(\tau)))} \nabla \tau
$$

Hence we get the desired result.

The delta derivative version of Lemma 3.1 was proved by Ferreira and Torres [11]. By using these results, we proved the following theorem.

Theorem 3.2 Assume that $u \in C_{\mathrm{ld}}\left([a, b]_{\mathbb{T}}, \mathbb{R}_{0}^{+}\right), c \in C_{\mathrm{ld}}\left([a, b]_{\mathbb{T}}, \mathbb{R}^{+}\right)$is nondecreasing, $\Phi \in$ $C\left(\mathbb{R}_{0}^{+}, \mathbb{R}_{0}^{+}\right)$is a strictly increasing function such that

$$
\lim _{x \rightarrow \infty} \Phi(x)=\infty
$$

Let $f(t, \xi), h(t, \xi) \in C_{\mathrm{ld}}\left([a, b]_{\mathbb{T}} \times[a, b]_{\mathbb{T}_{\kappa}}, \mathbb{R}_{0}^{+}\right)$and $g(t, \xi) \in C_{\mathrm{ld}}\left([a, b]_{\mathbb{T}_{\kappa}} \times[a, b]_{\mathbb{T}^{2}}, \mathbb{R}_{0}^{+}\right)$be nondecreasing in $t$ for every fixed $\xi$. Further, let $w, \phi, \Psi \in C\left(\mathbb{R}_{0}^{+}, \mathbb{R}_{0}^{+}\right)$be nondecreasing such that $\{w, \phi, \Psi\}(x)>0$ for every $x>0$. Assume that the following function

$$
F(x)=\int_{x_{0}}^{x} \frac{d s}{M \circ \Phi^{-1}(s)}
$$

with $x>c(a)>x_{0} \geq 0$ if $\int_{0}^{x} \frac{d s}{M \circ \Phi^{-1}(s)}<\infty$ and $x>c(a)>x_{0}>0$ if $\int_{0}^{x} \frac{d s}{M \circ \Phi^{-1}(s)}=\infty$; $\lim _{x \rightarrow \infty} F(x)=\infty$, where $M(x)=\max \{\phi(x), \Psi(x)\}$ on $\mathbb{R}_{0}^{+}$.

Also assume that the function

$$
P(r)=\int_{r_{0}}^{r} \frac{d s}{w(s)}
$$

where $r \geq 0, r_{0} \geq 0$ if $\int_{0}^{r} \frac{d s}{w(s)}<\infty$ and $r>0, r_{0}>0$ if $\int_{0}^{r} \frac{d s}{w(s)}=\infty$; $\lim _{r \rightarrow \infty} P(r)=\infty$.

Then there exist fixed constants $\alpha, \beta>0$ such that $\Phi^{-1}\left[F^{-1}(x)\right] \leq x$ for all $x \geq 0$, then

$$
\Phi[u(t)] \leq c(t)+\int_{a}^{t}\left(f(t, s) \phi[u(s)]\left[u(s)+\int_{a}^{s} g(s, \xi) w[u(s)] \nabla \xi\right]+h(t, s) \Psi[u(s)]\right) \nabla s
$$


for $t \in[a, b]_{\mathbb{T}}$ implies

$$
\begin{aligned}
u(t) \leq & \Phi^{-1}\left[F ^ { - 1 } \left(K(t)+\alpha \int_{a}^{t}\left(f(t, s) G^{-1}[G(K(t))\right.\right.\right. \\
& \left.\left.\left.\left.+\beta \int_{a}^{s} \max \{\alpha f(t, \xi), g(t, \xi)\} \nabla \xi\right]\right) \nabla s\right)\right] .
\end{aligned}
$$

Here $K(t):=F[c(t)]+\alpha \int_{a}^{t} h(t, s) \nabla s$ and $G(r)=\int_{r_{0}}^{r} \frac{d s}{w(s)+s}$.

Proof If $t=a$, then, obviously, theorem holds. Let us fix an arbitrary number $t_{0} \in(a, b]_{\mathbb{T}}$, we define $z(t)$ on $\left[a, t_{0}\right]_{\mathbb{T}}$ such that

$$
z(t)=c\left(t_{0}\right)+\int_{a}^{t}\left(f\left(t_{0}, s\right) \phi(u(s))\left[u(s)+\int_{a}^{s} g\left(t_{0}, \xi\right) w(u(\xi)) \nabla \xi\right]+h\left(t_{0}, s\right) \Psi[u(s)]\right) \nabla s .
$$

Since $\Phi(u(t)) \leq z(t)$, then $u(t) \leq \Phi^{-1}(z(t))$, so we have

$$
\frac{z^{\nabla}(t)}{M\left[\Phi^{-1}(z(t))\right]} \leq f\left(t_{0}, t\right)\left[\Phi^{-1}(z(t))+\int_{a}^{t} g\left(t_{0}, \xi\right) w\left[\Phi^{-1}(z(\xi))\right] \nabla \xi\right]+h\left(t_{0}, t\right)
$$

Define $\check{z}(t)$ on $[a, b]_{\mathbb{T}}$ such that

$$
\check{z}(t)=c(b)+\int_{a}^{t}\left(f(b, s) \phi[u(s)]\left[u(s)+\int_{a}^{s} g(b, \xi) w[u(\xi)] \nabla \xi\right]+h(b, s) \Psi[u(s)]\right) \nabla s .
$$

It is obvious that $z(t) \leq \check{z}(t)$ on $\left[a, t_{0}\right]_{\mathbb{T}}$. Using $\check{z}(t)$ we define $\alpha$ such that

$$
\alpha=\max _{t \in\left[a, t_{0}\right]_{T}} \frac{M \circ \Phi^{-1} \circ \check{z}(t)}{M \circ \Phi^{-1} \circ z(a)}>0 .
$$

From the definition of the functions $\check{z}(t), z(t), M, \Phi^{-1}$ and by Theorem 1.65 from [12] $\alpha$ exists.

Multiply (6) by $\alpha$ and use Lemma 3.2 to get

$$
\begin{aligned}
z(t) \leq & F^{-1}\left[F\left(c\left(t_{0}\right)\right)+\alpha \int_{a}^{t}\left(f\left(t_{0}, s\right)\left[\Phi^{-1}(z(s))+\int_{a}^{s} g\left(t_{0}, \xi\right) w\left[\Phi^{-1}(z(\xi))\right] \nabla \xi\right]\right) \nabla s\right. \\
& \left.+\alpha \int_{a}^{t_{0}} h\left(t_{0}, s\right) \nabla s\right]=F^{-1}(y(t)),
\end{aligned}
$$

where

$$
\begin{aligned}
y(t):= & F\left(c\left(t_{0}\right)\right)+\alpha \int_{a}^{t}\left(f\left(t_{0}, s\right)\left[\Phi^{-1}(z(s))+\int_{a}^{s} g\left(t_{0}, \xi\right) w\left[\Phi^{-1}(z(\xi))\right] \nabla \xi\right]\right) \nabla s \\
& +\alpha \int_{a}^{t_{0}} h\left(t_{0}, s\right) \nabla s .
\end{aligned}
$$

By differentiating the function $y(t)$ and using $\Phi^{-1}\left(F^{-1}(y(t))\right) \leq y(t)$, we obtain $y^{\nabla}(t) \leq$ $\alpha f\left(t_{0}, t\right)\left[y(t)+\int_{a}^{t} g\left(t_{0}, \xi\right) w[y(\xi)] \nabla \xi\right]$. Let us define $\Omega(t)=y(t)+\int_{a}^{t} g\left(t_{0}, \xi\right) w[y(\xi)] \nabla \xi$, so 
$\Omega(t) \geq y(t)$. Then

$$
\frac{\Omega^{\nabla}(t)}{\Omega(t)+w[\Omega(t)]} \leq \max \left\{\alpha f\left(t_{0}, t\right), g\left(t_{0}, t\right)\right\} .
$$

Let us define $\hat{y}(t)$ and $\hat{\Omega}(t)$ on $[a, b]_{\mathbb{T}}$ such that

$$
\begin{aligned}
& \hat{y}(t)=K(b)+\alpha \int_{a}^{t}\left(f(b, s)\left[\Phi^{-1}(z(s))+\int_{a}^{s} g(b, \xi) w\left[\Phi^{-1}(z(\xi)) \nabla \xi\right]\right]\right) \nabla s, \\
& \hat{\Omega}(t)=\hat{y}(t)+\int_{a}^{t} g(b, \xi) w[\hat{y}(\xi)] \nabla \xi .
\end{aligned}
$$

It is obvious that $y(t) \leq \hat{y}(t)$ and $\Omega(t) \leq \hat{\Omega}(t)$ on $\left[a, t_{0}\right]_{\mathbb{T}}$. Using $\hat{\Omega}(t)$ we define $\beta$ such that

$$
\beta=\max _{t \in\left[a, t_{0}\right]_{\mathbb{T}}} \frac{\hat{\Omega}(t)+w(\hat{\Omega}(t))}{\Omega(a)+w(\Omega(a))}>0 .
$$

Again from the definition of the functions $\Omega(t), y(t), \hat{y}(t), \hat{\Omega}(t), w(\hat{\Omega}(t))$ and by Theorem 1.65 from [12] $\beta$ exists, where

$$
\beta=\max _{t \in\left[a, t_{0}\right]_{T}} \frac{\hat{\Omega}(t)+w(\hat{\Omega}(t))}{\Omega(a)+w(\Omega(a))} \geq \frac{\Omega(t)+w(\Omega(t))}{\Omega(\rho(t))+w(\Omega(\rho(t)))} .
$$

If we multiply (7) by $\beta$ and use Lemma 3.2, we have

$$
\Omega(t)=G^{-1}\left[G\left(K\left(t_{0}\right)\right)+\beta \int_{a}^{t} \max \left\{\alpha f\left(t_{0}, s\right), g\left(t_{0}, s\right)\right\} \nabla s\right] .
$$

Since $y^{\nabla}(t) \leq \alpha f\left(t_{0}, t\right) \Omega(t)$, then $y(t) \leq K\left(t_{0}\right)+\alpha \int_{a}^{t} f\left(t_{0}, s\right) \Omega(s) \nabla s$. Also using the information $u(t) \leq \Phi^{-1}(z(t)) \leq \Phi^{-1}\left(F^{-1}(y(t))\right)$, we get

$$
\begin{aligned}
u(t) \leq & \Phi^{-1}\left[F ^ { - 1 } \left(K\left(t_{0}\right)+\alpha \int_{a}^{t}\left(f ( t _ { 0 } , s ) G ^ { - 1 } \left[G\left(K\left(t_{0}\right)\right)\right.\right.\right.\right. \\
& \left.\left.\left.\left.+\beta \int_{a}^{s} \max \left\{\alpha f\left(t_{0}, \xi\right), g\left(t_{0}, \xi\right)\right\} \nabla \xi\right]\right) \nabla s\right)\right] .
\end{aligned}
$$

Since $t_{0}$ is arbitrary, we can set $t=t_{0}$ in the above inequality, and we get the desired result.

Remark 3.1 The function $G(r)$ defined above satisfies $\lim _{r \rightarrow \infty} G(r)=\infty$ by Constantin [13]. This was discussed in [14-16].

Example 3.3 If we take $\phi(x)=\psi(x)=\Phi^{\prime}(x)$ with $\Phi^{\prime}$ being nondecreasing, then $M(x)=$ $\Phi^{\prime}(x)$, and this implies

$$
F(x)=\int_{x_{0}}^{x} \frac{1}{\Phi^{\prime} \circ \Phi^{-1}(s)} d s=\Phi^{-1}(x)-\Phi^{-1}\left(x_{0}\right) .
$$

Choose $x_{0}=\Phi(0) \geq 0$. Then $\Phi^{-1}(\Phi(0))=0$, hence $F(x)=\Phi^{-1}(x)$. For the particular case $\mathbb{T}=\mathbb{Z}$, an application of Theorem 3.2 gives Theorem 3.1. For the particular case $\mathbb{T}=\mathbb{R}$ and 
$\alpha=\beta=1$, an application of Theorem 3.2 gives the generalization of Constantin's inequality which is done by Yang and Tan [4].

Example 3.4 Let us take $\mathbb{T}=h \mathbb{Z}$ such that $x, t \in[h, M h], h(x, t)=H(x-t), g(x, t)=f(t, x)=$ $0, \Phi(x)=x^{2}, \phi(x)=0, \psi(x)=\frac{x}{2}$ defined for $x \geq 0$ and $c(n)=L, L \geq 0$ is constant. Then

$$
M(x)=\max \left\{\frac{x}{2}, 0\right\}=\frac{x}{2} .
$$

If we set $x_{0}=0$, we obtain

$$
F(x)=\int_{0}^{x} \frac{1}{\frac{\sqrt{s}}{2}} d s=4 \sqrt{x}
$$

Thus, $\lim _{x \rightarrow \infty} F(x)=\infty . F^{-1}(x)=\left(\frac{x}{4}\right)^{2}$ and $\Phi^{-1}\left(F^{-1}(x)\right)=\left(\frac{x}{4}\right) \leq x, \forall x \geq 0$.

If we get the equality, we have

$$
u^{2}(h n)=L+\sum_{t=1}^{n} h H(h n-h t) \frac{u(h t)}{2} .
$$

By letting $n=1,2$, we get

$$
\begin{aligned}
& u(h)=\sqrt{L+h H(0) \frac{u(h)}{2}}, \\
& u(2 h)=\sqrt{L+h H(h) \frac{u(h)}{2}+h H(0) \frac{u(2 h)}{2}} .
\end{aligned}
$$

If we apply Theorem 3.2, we find an upper bound for $u(h n)$ as

$$
u(h n) \leq \sqrt{L}+\alpha \frac{\sum_{t=1}^{n} h H(h n-h t)}{4} .
$$

Here

$$
\alpha=\max _{n \in[1, M]}\left[1+\frac{H((M-n) h) \frac{u(n h)}{2}}{L+H((M-1) h) \frac{u(h)}{2}+H((M-2) h) \frac{u(2 h)}{2}+\cdots+H((M-n+1) h) \frac{u((n-1) h)}{2}}\right] .
$$

Lemma 3.3 Let $\mathbb{T}$ be a regulated time scale, $a, b \in \mathbb{T}$, and consider the time scale $[a, b]_{\mathbb{T}}$ such that $\sigma(a)=a$. Let $r \in C^{1}\left([a, b]_{\mathbb{T}}, \mathbb{R}\right)$ with $r^{\nabla}(t), r^{\Delta}(t) \geq 0$. Suppose that a function $g \in$ $C\left(\mathbb{R}_{0}^{+}, \mathbb{R}_{0}^{+}\right)$is positive and nondecreasing on $\mathbb{R}$.

Define $G(x)=\int_{x_{0}}^{x} \frac{d s}{g(s)}$, where $x \geq 0, x_{0} \geq 0$ if $G(x)=\int_{0}^{x} \frac{d s}{g(s)}<\infty$ and $x>0, x_{0}>0$ if $G(x)=$ $\int_{0}^{x} \frac{d s}{g(s)}=\infty$. Then, for each $t \in[a, b]_{\mathbb{T}}$, we have

$$
(G \circ r)(t) \leq 2(G \circ r)(a)+2 \int_{a}^{t} \frac{r^{\diamond \alpha}(s)}{g(r(\rho(s)))} .
$$

Proof By assumption we have

$$
\frac{1}{g\left(r(\rho(t))+h v(t) r^{\nabla}(t)\right)} \leq \frac{1}{g(r(\rho(t)))}
$$


and

$$
\frac{1}{g\left(r(t)+h \mu(t) r^{\Delta}(t)\right)} \leq \frac{1}{g(r(t))}
$$

For $\alpha \in(0,1)$, we get

$$
\begin{aligned}
& \alpha r^{\Delta}(t) \int_{0}^{1} \frac{d h}{g\left(r(t)+h \mu(t) r^{\Delta}(t)\right)}+(1-\alpha) r^{\nabla}(t) \int_{0}^{1} \frac{d h}{g\left(r(\rho(t))+h v(t) r^{\nabla}(t)\right)} \\
& \quad \leq \alpha \frac{r^{\Delta}(t)}{g(r(t))}+(1-\alpha) \frac{r^{\nabla}(t)}{g(r(\rho(t)))}
\end{aligned}
$$

and

$$
(G \circ r)^{\diamond_{\alpha}}(t) \leq \frac{r^{\diamond}(t)}{g(r(\rho(t)))}
$$

By using $[8,17]$ and the assumption on $a$, we have the desired result

$$
\frac{1}{2} G \circ r(t) \leq G \circ r(a)+\int_{a}^{t} \frac{r^{\diamond \alpha}(s)}{g(r(\rho(s)))}
$$

Theorem 3.3 $u \in C\left([a, b]_{\mathbb{T}}, \mathbb{R}_{+}\right)$satisfies for some $k>0$ such that

$$
u^{2}(t) \leq k^{2}+2 \int_{a}^{t}\left[f(s) u(s)\left\{u(s)+\int_{a}^{s} g(\tau) w(u(\tau)) \diamond_{\alpha} \tau\right\}+h(s) u(s)\right] \diamond_{\alpha} s .
$$

Here our time scale is regulated and $\sigma(a)=a$.

$\forall t \in[a, b]_{\mathbb{T}}, f, g, h \in C\left([a, b]_{\mathbb{T}}, \mathbb{R}_{+}^{0}\right), w(t) \in C\left(\mathbb{R}_{+}^{0}, \mathbb{R}_{+}^{0}\right)$ and $w(t)$ is nondecreasing, there exist fixed constants $m, c>0$ such that

$$
\begin{aligned}
u(t) \leq & 4 k+2 c \int_{a}^{t}\left(1-2 \alpha+2 \alpha^{2}\right) h(\tau)+\left(\alpha-\alpha^{2}\right) h(\rho(\tau))+\left(\alpha-\alpha^{2}\right) h(\sigma(\tau)) \diamond_{\alpha} \tau \\
& +2 \int_{a}^{t}\left[\left(2 c\left(1-2 \alpha+2 \alpha^{2}\right)^{2}+4 c\left(\alpha-\alpha^{2}\right)^{2}\right) f(s)\right. \\
& +4 c\left(1-2 \alpha+2 \alpha^{2}\right)\left(\alpha-\alpha^{2}\right) f(\rho(s))+4 c\left(1-2 \alpha+2 \alpha^{2}\right)\left(\alpha-\alpha^{2}\right) f(\sigma(s)) \\
& \left.+2 c\left(\alpha-\alpha^{2}\right)^{2} f\left(\sigma^{2}(s)\right)+2 c\left(\alpha-\alpha^{2}\right)^{2} f\left(\rho^{2}(s)\right)\right] \\
& \times\left[E ^ { - 1 } \left\{2 E \left(2 k+c \int_{a}^{t_{0}}\left(1-2 \alpha+2 \alpha^{2}\right) h(\tau)+\left(\alpha-\alpha^{2}\right) h(\rho(\tau))\right.\right.\right. \\
& \left.+\left(\alpha-\alpha^{2}\right) h(\sigma(\tau)) \diamond_{\alpha} \tau\right)+\int_{a}^{s}\left[\left(4 m c\left(1-2 \alpha+2 \alpha^{2}\right)^{2}+8 m c\left(\alpha-\alpha^{2}\right)^{2}\right) f\left(\sigma^{2}(\tau)\right)\right. \\
& +8 m c\left(1-2 \alpha+2 \alpha^{2}\right)\left(\alpha-\alpha^{2}\right) f(\sigma(\tau))+8 m c\left(1-2 \alpha+2 \alpha^{2}\right)\left(\alpha-\alpha^{2}\right) f\left(\sigma^{3}(\tau)\right) \\
& \left.+4 m c\left(\alpha-\alpha^{2}\right)^{2} f\left(\sigma^{4}(\tau)\right)+4 m c\left(\alpha-\alpha^{2}\right)^{2} f(\tau)\right] \diamond_{\alpha} \tau \\
& +\int_{a}^{s}\left[2\left(1-2 \alpha+2 \alpha^{2}\right) m g\left(\sigma^{2}(\tau)\right)+2\left(\alpha-\alpha^{2}\right) m g(\sigma(\tau))\right. \\
& \left.\left.\left.+2\left(\alpha-\alpha^{2}\right) m g\left(\sigma^{3}(\tau)\right)\right] \diamond_{\alpha} \tau\right\}\right] \diamond_{\alpha} s,
\end{aligned}
$$

where $E(r)=\int_{1}^{r} \frac{d s}{w(s)+s}, r>0$. 
Proof If we take $a=t$, then the inequality obviously holds true. Let $t_{0} \in(a, b]$ and define $z(t)$ in $\left[a, t_{0}\right]$ such that

$$
z(t)=k^{2}+2 \int_{a}^{t}\left[f(s) u(s)\left\{u(s)+\int_{a}^{s} g(\tau) w(u(\tau)) \diamond_{\alpha} \tau\right\}+h(s) u(s)\right] \diamond_{\alpha} s .
$$

Therefore, for $u(t) \leq \sqrt{z(t)}, t \in\left[a, t_{0}\right]_{\mathbb{T}}$. Since $z^{\Delta}(t), z^{\nabla}(t) \geq 0$, if we use [8], we obtain

$$
\begin{aligned}
0 \leq & z^{\diamond_{\alpha}}(t) \\
\leq & 2\left(1-2 \alpha+2 \alpha^{2}\right)\left[f(t) \sqrt{z(t)}\left\{\sqrt{z(t)}+\int_{a}^{t} g(\tau) w(\sqrt{z(\tau)}) \diamond_{\alpha} \tau\right\}+h(t) \sqrt{z(t)}\right] \\
& +2\left(\alpha-\alpha^{2}\right)\left[f(\rho(t)) \sqrt{z(\rho(t))}\left\{\sqrt{z(\rho(t))}+\int_{a}^{\rho(t)} g(\tau) w(\sqrt{z(\tau)}) \diamond_{\alpha} \tau\right\}\right. \\
& +h(\rho(t)) \sqrt{z(\rho(t))}] \\
& +2\left(\alpha-\alpha^{2}\right)\left[f(\sigma(t)) \sqrt{z(\sigma(t))}\left\{\sqrt{z(\sigma(t))}+\int_{a}^{\sigma(t)} g(\tau) w(\sqrt{z(\tau)}) \diamond_{\alpha} \tau\right\}\right. \\
& +h(\sigma(t)) \sqrt{z(\sigma(t))}] .
\end{aligned}
$$

Then $z(t)$ is nondecreasing. In other words, $z(\sigma(t)) \geq z(t) \geq z(\rho(t))$, so we have

$$
\begin{aligned}
\frac{z^{\diamond \alpha}(t)}{2 \sqrt{z(\sigma(t))} \leq} & \left(1-2 \alpha+2 \alpha^{2}\right)\left[f(t)\left\{\sqrt{z(t)}+\int_{a}^{t} g(\tau) w(\sqrt{z(\tau)}) \diamond_{\alpha} \tau\right\}+h(t)\right] \\
& +\left(\alpha-\alpha^{2}\right)\left[f(\rho(t))\left\{\sqrt{z(\rho(t))}+\int_{a}^{\rho(t)} g(\tau) w(\sqrt{z(\tau)}) \diamond_{\alpha} \tau\right\}\right. \\
& +h(\rho(t))] \\
& +\left(\alpha-\alpha^{2}\right)\left[f(\sigma(t))\left\{\sqrt{z(\sigma(t))}+\int_{a}^{\sigma(t)} g(\tau) w(\sqrt{z(\tau)}) \diamond_{\alpha} \tau\right\}\right. \\
& +h(\sigma(t))]
\end{aligned}
$$

Functions that compose $z(t)$ are from $C\left([a, b]_{\mathbb{T}}, \mathbb{R}_{+}^{0}\right)$, then they are regulated on $[a, b]_{\mathbb{T}}$, then $z(t)$ is bounded and $z(t)$ never takes zero. Therefore there exists $c$ such that $c=$ $\max _{t \in\left[a, t_{0}\right]_{\mathbb{T}}} \frac{\sqrt{z(\sigma(t))}}{\sqrt{z(\rho(t))}}$.

Multiply (8) by $c$ and obtain

$$
\begin{aligned}
\frac{z^{\diamond}(t)}{2 \sqrt{z(\rho(t))} \leq} & c\left(1-2 \alpha+2 \alpha^{2}\right)\left[f(t)\left\{\sqrt{z(t)}+\int_{a}^{t} g(\tau) w(\sqrt{z(\tau)}) \diamond_{\alpha} \tau\right\}+h(t)\right] \\
& +c\left(\alpha-\alpha^{2}\right)\left[f(\rho(t))\left\{\sqrt{z(\rho(t))}+\int_{a}^{\rho(t)} g(\tau) w(\sqrt{z(\tau)}) \diamond_{\alpha} \tau\right\}+h(\rho(t))\right] \\
& +c\left(\alpha-\alpha^{2}\right)\left[f(\sigma(t))\left\{\sqrt{z(\sigma(t))}+\int_{a}^{\sigma(t)} g(\tau) w(\sqrt{z(\tau)}) \diamond_{\alpha} \tau\right\}+h(\sigma(t))\right] .
\end{aligned}
$$


Now we use Lemma 3.3 and we get

$$
\begin{aligned}
\sqrt{z(t)} \leq & 2 k+2 c\left(1-2 \alpha+2 \alpha^{2}\right) \int_{a}^{t}\left[f(s)\left\{\sqrt{z(s)}+\int_{a}^{s} g(\tau) w(\sqrt{z(\tau)}) \diamond_{\alpha} \tau\right\}\right] \diamond_{\alpha} s \\
& +2 c\left(\alpha-\alpha^{2}\right) \int_{a}^{t}\left[f(\rho(s))\left\{\sqrt{z(\rho(s))}+\int_{a}^{\rho(s)} g(\tau) w(\sqrt{z(\tau)}) \diamond_{\alpha} \tau\right\}\right] \diamond_{\alpha} s \\
& +2 c\left(\alpha-\alpha^{2}\right) \int_{a}^{t}\left[f(\sigma(s))\left\{\sqrt{z(\sigma(s))}+\int_{a}^{\sigma(s)} g(\tau) w(\sqrt{z(\tau)}) \diamond_{\alpha} \tau\right\}\right] \diamond_{\alpha} s \\
& +\int_{a}^{t_{0}}\left(1-2 \alpha+2 \alpha^{2}\right) h(s)+\left(\alpha-\alpha^{2}\right) h(\rho(s))+\left(\alpha-\alpha^{2}\right) h(\sigma(s)) \diamond_{\alpha} s .
\end{aligned}
$$

Let us say the right-hand side of the above inequality is $V(t)$, then $\sqrt{z(t)} \leq V(t)$. So we get

$$
\begin{aligned}
V^{\diamond_{\alpha}}(t) \leq & \left(2 c\left(1-2 \alpha+2 \alpha^{2}\right)^{2}+4 c\left(\alpha-\alpha^{2}\right)^{2}\right)\left[f(t)\left\{V(t)+\int_{a}^{t} g(\tau) w(V(\tau)) \diamond_{\alpha} \tau\right\}\right] \\
& +4 c\left(1-2 \alpha+2 \alpha^{2}\right)\left(\alpha-\alpha^{2}\right)\left[f(\rho(t))\left\{V(\rho(t))+\int_{a}^{\rho(s)} g(\tau) w(V(\tau)) \diamond_{\alpha} \tau\right\}\right] \\
& +4 c\left(1-2 \alpha+2 \alpha^{2}\right)\left(\alpha-\alpha^{2}\right)\left[f(\sigma(t))\left\{V(\sigma(t))+\int_{a}^{\sigma(t)} g(\tau) w(V(\tau)) \diamond_{\alpha} \tau\right\}\right] \\
& +2 c\left(\alpha-\alpha^{2}\right)^{2}\left[f\left(\rho^{2}(t)\right)\left\{V\left(\rho^{2}(t)\right)+\int_{a}^{\rho^{2}(t)} g(\tau) w(V(\tau)) \diamond_{\alpha} \tau\right\}\right] \\
& +2 c\left(\alpha-\alpha^{2}\right)^{2}\left[f\left(\sigma^{2}(t)\right)\left\{V\left(\sigma^{2}(t)\right)+\int_{a}^{\sigma^{2}(t)} g(\tau) w(V(\tau)) \diamond_{\alpha} \tau\right\}\right] .
\end{aligned}
$$

Here $V^{\diamond_{\alpha}}(t) \geq 0$. Similarly, if we take the delta and nabla derivative of $V(t)$, we also see that $V^{\Delta}(t), V^{\nabla}(t) \geq 0$.

Therefore $V\left(\sigma^{2}(t)\right) \geq V(\sigma(t)) \geq V(t) \geq V(\rho(t)) \geq V\left(\rho^{2}(t)\right)$. Then

$$
\begin{aligned}
V^{\diamond_{\alpha}}(t) \leq & {\left[\left(2 c\left(1-2 \alpha+2 \alpha^{2}\right)^{2}+4 c\left(\alpha-\alpha^{2}\right)^{2}\right) f(t)+4 c\left(1-2 \alpha+2 \alpha^{2}\right)\left(\alpha-\alpha^{2}\right) f(\rho(t))\right.} \\
& +4 c\left(1-2 \alpha+2 \alpha^{2}\right)\left(\alpha-\alpha^{2}\right) f(\sigma(t))+2 c\left(\alpha-\alpha^{2}\right)^{2} f\left(\sigma^{2}(t)\right) \\
& \left.+2 c\left(\alpha-\alpha^{2}\right)^{2} f\left(\rho^{2}(t)\right)\right]\left\{V\left(\sigma^{2}(t)\right)+\int_{a}^{\sigma^{2}(t)} g(\tau) w(V(\tau)) \diamond_{\alpha} \tau\right\} .
\end{aligned}
$$

Let us take $V\left(\sigma^{2}(t)\right)+\int_{a}^{\sigma^{2}(t)} g(\tau) w(V(\tau)) \diamond_{\alpha} \tau=\Omega(t)$. Taking the nabla derivative of $\Omega(t)$ and using (9), we have

$$
\begin{aligned}
\Omega^{\diamond}(t) \leq & {\left[\left(2 c\left(1-2 \alpha+2 \alpha^{2}\right)^{2}+4 c\left(\alpha-\alpha^{2}\right)^{2}\right) f\left(\sigma^{2}(t)\right)\right.} \\
& +4 c\left(1-2 \alpha+2 \alpha^{2}\right)\left(\alpha-\alpha^{2}\right) f(\sigma(t)) \\
& +4 c\left(1-2 \alpha+2 \alpha^{2}\right)\left(\alpha-\alpha^{2}\right) f\left(\sigma^{3}(t)\right) \\
& \left.+2 c\left(\alpha-\alpha^{2}\right)^{2} f\left(\sigma^{4}(t)\right)+2 c\left(\alpha-\alpha^{2}\right)^{2} f(t)\right] \Omega(t) \\
& +\left(1-2 \alpha+2 \alpha^{2}\right) g\left(\sigma^{2}(t)\right) w\left(V\left(\sigma^{2}(t)\right)\right)+\left(\alpha-\alpha^{2}\right) g(\sigma(t)) w(V(\sigma(t))) \\
& +\left(\alpha-\alpha^{2}\right) g\left(\sigma^{3}(t)\right) w\left(V\left(\sigma^{3}(t)\right)\right)
\end{aligned}
$$


$\Omega^{\diamond_{\alpha}}(t) \geq 0$. Similarly, $\Omega^{\Delta}(t), \Omega^{\nabla}(t) \geq 0$. It is obvious that $V\left(\sigma^{2}(t)\right) \leq \Omega(t)$ and since $w$ is nondecreasing, we also have $w(\Omega(\sigma(t))) \geq w(\Omega(t)) \geq w(\Omega(\rho(t)))$. Therefore $w(\Omega(\sigma(t))) \geq$ $w\left(V\left(\sigma^{3}(t)\right)\right)$,

$$
\begin{aligned}
& \frac{\Omega^{\diamond}(t)}{\Omega(t)+w(\Omega(\sigma(t)))} \\
& \leq\left[\left(2 c\left(1-2 \alpha+2 \alpha^{2}\right)^{2}+4 c\left(\alpha-\alpha^{2}\right)^{2}\right) f\left(\sigma^{2}(t)\right)+4 c\left(1-2 \alpha+2 \alpha^{2}\right)\left(\alpha-\alpha^{2}\right) f(\sigma(t))\right. \\
& \left.\quad+4 c\left(1-2 \alpha+2 \alpha^{2}\right)\left(\alpha-\alpha^{2}\right) f\left(\sigma^{3}(t)\right)+2 c\left(\alpha-\alpha^{2}\right)^{2} f\left(\sigma^{4}(t)\right)+2 c\left(\alpha-\alpha^{2}\right)^{2} f(t)\right] \\
& \quad+\left[\left(1-2 \alpha+2 \alpha^{2}\right) g\left(\sigma^{2}(t)\right)+\left(\alpha-\alpha^{2}\right) g(\sigma(t))+\left(\alpha-\alpha^{2}\right) g\left(\sigma^{3}(t)\right)\right] .
\end{aligned}
$$

By using the same information while finding the existence of $c$, there exists $m$ such that

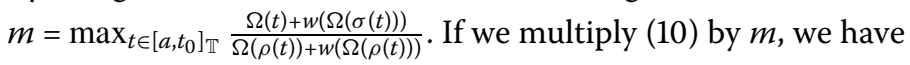

$$
\begin{aligned}
& \frac{\Omega^{\diamond}(t)}{\Omega(\rho(t))+w(\Omega(\rho(t)))} \\
& \leq\left[\left(2 m c\left(1-2 \alpha+2 \alpha^{2}\right)^{2}+4 m c\left(\alpha-\alpha^{2}\right)^{2}\right) f\left(\sigma^{2}(t)\right)\right. \\
& \quad+4 m c\left(1-2 \alpha+2 \alpha^{2}\right)\left(\alpha-\alpha^{2}\right) f(\sigma(t)) \\
& \quad+4 m c\left(1-2 \alpha+2 \alpha^{2}\right)\left(\alpha-\alpha^{2}\right) f\left(\sigma^{3}(t)\right) \\
& \left.\quad+2 m c\left(\alpha-\alpha^{2}\right)^{2} f\left(\sigma^{4}(t)\right)+2 m c\left(\alpha-\alpha^{2}\right)^{2} f(t)\right] \\
& \quad+\left[\left(1-2 \alpha+2 \alpha^{2}\right) m g\left(\sigma^{2}(t)\right)+\left(\alpha-\alpha^{2}\right) m g(\sigma(t))+\left(\alpha-\alpha^{2}\right) m g\left(\sigma^{3}(t)\right)\right] .
\end{aligned}
$$

Now we use Lemma 3.3 and get

$$
\begin{aligned}
\Omega(t) \leq & E^{-1}\left\{2 E \left(2 k+c \int_{a}^{t_{0}}\left(1-2 \alpha+2 \alpha^{2}\right) h(s)\right.\right. \\
& \left.+\left(\alpha-\alpha^{2}\right) h(\rho(s))+\left(\alpha-\alpha^{2}\right) h(\sigma(s)) \diamond_{\alpha} s\right) \\
& +\int_{a}^{t}\left[\left(4 m c\left(1-2 \alpha+2 \alpha^{2}\right)^{2}+8 m c\left(\alpha-\alpha^{2}\right)^{2}\right) f\left(\sigma^{2}(s)\right)\right. \\
& +8 m c\left(1-2 \alpha+2 \alpha^{2}\right)\left(\alpha-\alpha^{2}\right) f(\sigma(s))+8 m c\left(1-2 \alpha+2 \alpha^{2}\right)\left(\alpha-\alpha^{2}\right) f\left(\sigma^{3}(s)\right) \\
& \left.+4 m c\left(\alpha-\alpha^{2}\right)^{2} f\left(\sigma^{4}(s)\right)+4 m c\left(\alpha-\alpha^{2}\right)^{2} f(s)\right] \diamond_{\alpha} s \\
& +\int_{a}^{t}\left[2\left(1-2 \alpha+2 \alpha^{2}\right) m g\left(\sigma^{2}(s)\right)+2\left(\alpha-\alpha^{2}\right) m g(\sigma(s))\right. \\
& \left.\left.+2\left(\alpha-\alpha^{2}\right) m g\left(\sigma^{3}(s)\right)\right] \diamond_{\alpha} s\right\} .
\end{aligned}
$$

Then we have

$$
\begin{aligned}
V^{\diamond_{\alpha}}(t) \leq & {\left[\left(2 c\left(1-2 \alpha+2 \alpha^{2}\right)^{2}+4 c\left(\alpha-\alpha^{2}\right)^{2}\right) f(t)+4 c\left(1-2 \alpha+2 \alpha^{2}\right)\left(\alpha-\alpha^{2}\right) f(\rho(t))\right.} \\
& +4 c\left(1-2 \alpha+2 \alpha^{2}\right)\left(\alpha-\alpha^{2}\right) f(\sigma(t))+2 c\left(\alpha-\alpha^{2}\right)^{2} f\left(\sigma^{2}(t)\right) \\
& \left.+2 c\left(\alpha-\alpha^{2}\right)^{2} f\left(\rho^{2}(t)\right)\right] E^{-1}\left\{2 E \left(2 k+c \int_{a}^{t_{0}}\left(1-2 \alpha+2 \alpha^{2}\right) h(s)\right.\right.
\end{aligned}
$$




$$
\begin{aligned}
& \left.+\left(\alpha-\alpha^{2}\right) h(\rho(s))+\left(\alpha-\alpha^{2}\right) h(\sigma(s)) \diamond_{\alpha} s\right) \\
& +\int_{a}^{t}\left[\left(4 m c\left(1-2 \alpha+2 \alpha^{2}\right)^{2}+8 m c\left(\alpha-\alpha^{2}\right)^{2}\right) f\left(\sigma^{2}(s)\right)\right. \\
& +8 m c\left(1-2 \alpha+2 \alpha^{2}\right)\left(\alpha-\alpha^{2}\right) f(\sigma(s))+8 m c\left(1-2 \alpha+2 \alpha^{2}\right)\left(\alpha-\alpha^{2}\right) f\left(\sigma^{3}(s)\right) \\
& \left.+4 m c\left(\alpha-\alpha^{2}\right)^{2} f\left(\sigma^{4}(s)\right)+4 m c\left(\alpha-\alpha^{2}\right)^{2} f(s)\right] \diamond_{\alpha} s \\
& +\int_{a}^{t}\left[2\left(1-2 \alpha+2 \alpha^{2}\right) m g\left(\sigma^{2}(s)\right)+2\left(\alpha-\alpha^{2}\right) m g(\sigma(s))\right. \\
& \left.\left.+2\left(\alpha-\alpha^{2}\right) m g\left(\sigma^{3}(s)\right)\right] \diamond_{\alpha} s\right\}
\end{aligned}
$$

Using [8] we obtain

$$
\begin{aligned}
\frac{1}{2} V(t) \leq & k+c \int_{a}^{t_{0}}\left(1-2 \alpha+2 \alpha^{2}\right) h(\tau)+\left(\alpha-\alpha^{2}\right) h(\rho(\tau))+\left(\alpha-\alpha^{2}\right) h(\sigma(\tau)) \diamond_{\alpha} \tau \\
& +\int_{a}^{t}\left[\left(2 c\left(1-2 \alpha+2 \alpha^{2}\right)^{2}+4 c\left(\alpha-\alpha^{2}\right)^{2}\right) f(s)\right. \\
& +4 c\left(1-2 \alpha+2 \alpha^{2}\right)\left(\alpha-\alpha^{2}\right) f(\rho(s))+4 c\left(1-2 \alpha+2 \alpha^{2}\right)\left(\alpha-\alpha^{2}\right) f(\sigma(s)) \\
& \left.+2 c\left(\alpha-\alpha^{2}\right)^{2} f\left(\sigma^{2}(s)\right)+2 c\left(\alpha-\alpha^{2}\right)^{2} f\left(\rho^{2}(s)\right)\right] \\
& \times\left[E ^ { - 1 } \left\{2 E \left(2 k+c \int_{a}^{t_{0}}\left(1-2 \alpha+2 \alpha^{2}\right) h(\tau)\right.\right.\right. \\
& \left.+\left(\alpha-\alpha^{2}\right) h(\rho(\tau))+\left(\alpha-\alpha^{2}\right) h(\sigma(\tau)) \diamond_{\alpha} \tau\right) \\
& +\int_{a}^{s}\left[\left(4 m c\left(1-2 \alpha+2 \alpha^{2}\right)^{2}+8 m c\left(\alpha-\alpha^{2}\right)^{2}\right) f\left(\sigma^{2}(\tau)\right)\right. \\
& +8 m c\left(1-2 \alpha+2 \alpha^{2}\right)\left(\alpha-\alpha^{2}\right) f(\sigma(\tau))+8 m c\left(1-2 \alpha+2 \alpha^{2}\right)\left(\alpha-\alpha^{2}\right) f\left(\sigma^{3}(\tau)\right) \\
& \left.+4 m c\left(\alpha-\alpha^{2}\right)^{2} f\left(\sigma^{4}(\tau)\right)+4 m c\left(\alpha-\alpha^{2}\right)^{2} f(\tau)\right] \diamond_{\alpha} \tau \\
& +\int_{a}^{s}\left[2\left(1-2 \alpha+2 \alpha^{2}\right) m g\left(\sigma^{2}(\tau)\right)+2\left(\alpha-\alpha^{2}\right) m g(\sigma(\tau))\right. \\
& \left.\left.\left.+2\left(\alpha-\alpha^{2}\right) m g\left(\sigma^{3}(\tau)\right)\right] \diamond_{\alpha} \tau\right\}\right] \diamond_{\alpha} s .
\end{aligned}
$$

Since $u(t) \leq \sqrt{z(t)} \leq V(t)$, we get the desired result.

Example 3.5 If we choose $\alpha=1, \mathbb{T}=\mathbb{R}, m=c=1 / 2$, then Theorem 3.3 becomes Theorem 1.1.

Example 3.6 Let $\mathbb{T}=\{0\} \cup\left\{\frac{1}{n+1}: n \in \mathbb{N}\right\} \cup \mathbb{N}$. Here we assume that $\mathbb{N}$ starts with 1 . It is obvious that our time scales is regulated and $0=\sigma(0)$. Let us choose $M \in \mathbb{N}$ and investigate the following equality on the time scales $[0, M]_{\mathbb{T}}$ :

$$
u^{2}(t)=k^{2}+2 \int_{0}^{t} h(s) u(s) \diamond_{\alpha} s
$$


$h(t)=0$ if $t \in\{0\} \cup\left\{\frac{1}{n+1}: n \in \mathbb{N}\right\}$ and $h(t)=P(t)$ if $t \in \mathbb{N}$. Therefore $u(t)=k$ if $t \in\{0\} \cup\left\{\frac{1}{n+1}:\right.$ $n \in \mathbb{N}\}, u^{2}(n)=k^{2}+P(1) u(1)+P(2) u(2)+\cdots+P(n-1) u(n-1)+(1-\alpha) P(n) u(n)$ if $n \in[0, M]$.

If we apply Theorem 3.3 , we get the bound for $u(t)$ such that

$$
\begin{aligned}
u(t) \leq & 4 k+2 c \sum_{\tau=1}^{t-1}\left(1-2 \alpha+2 \alpha^{2}\right) P(\tau)+\left(\alpha-\alpha^{2}\right) P(\tau-1)+\left(\alpha-\alpha^{2}\right) P(\tau+1) \\
& +2 c(1-\alpha)\left[\left(1-2 \alpha+2 \alpha^{2}\right) P(t)+\left(\alpha-\alpha^{2}\right) P(t-1)+\left(\alpha-\alpha^{2}\right) P(t+1)\right]
\end{aligned}
$$

where

$$
c=\max _{n \in[1, M]} \sqrt{1+\frac{2 P(n-1) u(n-1)+2(1-\alpha) P(n) u(n)}{k^{2}+2 P(1) u(1)+2 P(2) u(2)+\cdots+2 P(n-2) u(n-2)}} .
$$

If we take $\alpha=\frac{1}{2}, k^{2}=2$, then for the equation $u^{2}(t)=2+2 \int_{0}^{t} h(s) u(s) \diamond_{\alpha} s$, where $h(s)=0$, if $t \in\{0\} \cup\left\{\frac{1}{n+1}: n \in \mathbb{N}\right\}$ and $h(s)=1$ if $t \in \mathbb{N}$, then $c=\frac{1}{2}$ and $u(t)$ is bound with $u(t) \leq \sqrt{2}$ if $t \in\{0\} \cup\left\{\frac{1}{n+1}: n \in \mathbb{N}\right\}$ and $u(n) \leq 4 \sqrt{2}+n-\frac{1}{2}$ if $n \in \mathbb{N}$.

\section{Competing interests}

The authors declare that they have no competing interests.

\section{Authors' contributions}

BK gave the first idea that is studying about the nabla and diamond-alpha version of Constantin's inequality, and she also made the literature review. AFG designed the pattern of the study and all findings were controlled by her in each step with BK. NNP participated in the contribution in the mathematical analysis part. AFG and NNP drafted the manuscript together. BK gave the final approval. All authors read and approved the final manuscript.

\section{Author details}

${ }^{1}$ Department of Mathematics, Faculty of Science, Ankara University, Ankara, Turkey. ${ }^{2}$ Department of Mathematics and Computer Science, Çankaya University, Ankara, 06810, Turkey. ${ }^{3}$ Department of Mathematics, Middle East Technical University, Ankara, 06810, Turkey.

Received: 11 November 2014 Accepted: 22 April 2015 Published online: 28 May 2015

\section{References}

1. Ou-lang, L: The boundedness of solutions of linear differential equations $y^{\prime \prime}+A(t) y=0$. Shuxue Jinzhan 3,409-418 (1957)

2. Pachpatte, BG: Some new inequalities related to certain inequalities in the theory of differential equations. J. Math. Anal. Appl. 189(1), 128-144 (1995)

3. Constantin, A: Topological transversality: application to an integrodifferential equation. J. Math. Anal. Appl. 197(3), 855-863 (1996)

4. Yang, EH, Tan, MC: A generalization of Constantin's integral inequality and its discrete analogue. J. Inequal. Pure Appl. Math. 8(2), Article 57 (2007)

5. Agarwal, RP, Bohner, M: Basic calculus on time scales and some of its applications. Results Math. 35(1-2), 3-22 (1999)

6. Anderson, DR: Taylor polynomials for nabla dynamic equations on time scales. Panam. Math. J. 12(4), 17-27 (2002)

7. Bohner, M, Peterson, A: Advances in Dynamic Equations on Time Scales. Birkhäuser Boston, Boston (2003)

8. Sheng, Q, Fadag, M, Henderson, J, Davis, JM: An exploration of combined dynamic derivatives on time scales and their applications. Nonlinear Anal., Real World Appl. 7, 395-413 (2006)

9. Ferreira, RAC: Constantin integral inequalities on time scales. Arch. Math. 93, 153-163 (2009)

10. Atici, FM, Biles, CD, Lebedinsky, A: An application of times scales to economics. Math. Comput. Model. 43(7-8), 718-726 (2006)

11. Ferreira, RAC, Torres, DFM: Generalizations of Gronwall Bihari inequalities on time scales. J. Differ. Equ. Appl. 15(6), 529-539 (2009)

12. Bohner, M, Peterson, A: Dynamic Equations on Times Scales: An Introduction with Applications. Birkhäuser, Basel (2001)

13. Constantin, A: Solutions globales d'équations différentielles perturbées. C. R. Acad. Sci. Paris 320, 1319-1322 (1995)

14. Bernfeld, SR: The extendability of solutions of perturbed scalar differential equations. Pac. J. Math. 42, 277-288 (1972)

15. Constantin, A: Global existence of solutions for perturbed differential equations. Ann. Mat. Pura Appl. (4) 168, 237-299 (1995)

16. Hara, T, Yoneyama, T, Sugie, J: Continuability of solutions of perturbed differential equations. Nonlinear Anal. 8(8), 963-975 (1984)

17. Atici, FM, Guseinov, GS: On Green's functions and positive solutions for boundary value problems on time scales. J. Comput. Appl. Math. 141(1-2), 75-99 (2002) 